Motrivivência Ano XXV, No 41, P. 281-289 Dez./2013

\title{
UM DIÁLOGO SOBRE A CULTURA CORPORAL E AS DIMENSÕES DOS CONTEÚDOS DENTRO DE UMA TEIA DE RELAÇÕES
}

Raquel Firmino Magalhães Barbosa'

\section{RESUMO}

Este artigo tem como objetivo buscar uma reflexão sobre a relação da cultura corporal e sua aplicação na Educação Física Escolar com as dimensões do conteúdo e os pilares da educação no processo de ensino-aprendizagem e na prática pedagógica. Questiona se o professor de Educação Física ao lidar com os conteúdos da cultura corporal teria o conhecimento imediato para desenvolver as capacidades nos educandos de uma maneira que encontrem sentido no que fazem? Verifica-se a importância de compreender os elementos do processo educacional e também seus objetivos e conteúdos como uma verdadeira teia educacional.

Palavras-chave: Cultura corporal; Dimensão dos conteúdos; Processo ensinoaprendizagem; Prática pedagógica.

1 Mestrado em Educação/ Universidade Federal de Mato Grosso. Cuiabá/Mato Grosso, Brasil.

E-mail: kekelfla@yahoo.com.br 
Dialogar com a cultura corporal é conhecer, compreender e explorar as manifestações que o nosso corpo construiu ao longo do tempo. A escola tem a possibilidade de promover vivências e explorar a história sobre as práticas corporais, adentrando em seus saberes, em suas origens e também em sua trajetória por meio da Educação Física, que abriu um leque para os elementos que compõe a cultura corporal, diversificando a construção do conhecimento.

Para nortear este estudo, partimos da seguinte problemática: o professor de Educação Física, ao lidar com os conteúdos da cultura corporal, teria o conhecimento imediato para desenvolver as capacidades nos educandos de uma maneira que encontrem sentido no que fazem?

Com o intuito de promover um diálogo sobre a cultura corporal e o seu sentido para o educando, este artigo tem como objetivo buscar uma reflexão sobre a relação da cultura corporal e sua aplicação na Educação Física Escolar com as dimensões do conteúdo e os pilares da educação no processo de ensino-aprendizagem e na prática pedagógica.

Este ensaio será composto por importantes teóricos e documentos que guiaram as reflexões aqui propostas com Daólio (2007), Geertz (2008) e Rangel (2008), Gonçalves (1997), através dos conceitos de cultura; Darido e Rangel (2005), com a discussão em torno da cultura corporal; Os Parâmetros Curriculares Nacionais (1997, 1999), com a educação, cultura corporal e a Educação Física; Lei de Diretrizes e Bases da Educação Nacional (1996), trazendo pontos importantes da legislação sobre a Educação Física; e Zabala (1998), com as dimensões do conteúdo. Todas essas referências ajudaram a enriquecer e integrar os pontos principais deste artigo.

Ao versar sobre a cultura corporal é preciso analisar alguns pontos essenciais para que possamos realizar uma reflexão sobre a aplicação e o entendimento sobre a mesma na escola. Passaremos pelos temas sobre o conceito de cultura até chegarmos na cultura corporal de movimento, e fazer uma relação com os pilares da educação e a classificação/dimensão dos conteúdos dentro da cultura corporal.

Ao conceituar cultura percebemos que ela é polissêmica, como veremos a seguir. De acordo com o dicionário Houaiss (2007), na acepção antropológica do termo, cultura se refere a um conjunto de padrões de comportamento, crenças, conhecimentos, costumes que distinguem um grupo social.

Na visão de Geertz (2008), podemos entender o conceito de cultura, como um sistema simbólico em que os indivíduos constroem sua própria história, ou seja, como um texto em que o ser humano está imerso e que precisa ser interpretado.

Segundo Daólio (2007), a cultura é o conceito essencial da Educação Física, pois todas as manifestações corporais humanas são geradas na dinâmica cultural, se expressando de modo diversificado e com significados próprios no contexto de grupos culturais específicos.

Para Rangel (2008), a cultura está além é de um conceito acadêmico, mas ligado vivências dos sujeitos, as suas formas de concepção do mundo, as particularidades e semelhanças construídas pelos educandos ao longo de seu processo histórico e social.

Já os Parâmetros Curriculares Nacionais abordam a cultura e a relevância da Educação Física para a construção do 
conhecimento, reforçando que a mesma "[...] precisa buscar sua identidade como área de estudo fundamental para a compreensão e entendimento do ser humano, enquanto produtor de cultura" (BRASIL, 1999, p. 156).

O corpo é expressão de cultura, logo, podemos concluir que o homem sempre foi produtor e reprodutor de cultura durante a história da humanidade. Assim, o professor de Educação Física trabalha com as manifestações culturais diretamente vinculadas ao corpo e ao movimento (DAÓLIO, 2007). A habilidade de produzir cultura bem como de se adaptar, de reconstruir e de resignificar seus conhecimentos ao longo do tempo, constituiu o que podemos chamar de cultura corporal de movimento, que foram introduzidas dentro da Educação Física (DARIDO \& RANGEL, 2005).

A conexão entre cultura, corpo e movimento são essenciais para o entendimento sobre a motricidade, sobre os fatos e comportamentos históricos, contemporâneos e culturais, compartilhados nas diferentes sociedades. Desta maneira, entende-se, de acordo com Soares (1992 apud DARIDO \& RANGEL, 2005, p. 26) que "[...] o conjunto desses fenômenos ou manifestações expressivas corporais tem sido denominado cultura corporal de movimento".

Esta concepção se preocupa com a contextualização das atividades expressivas e corporais durante as aulas, aproximando-se da realidade dos sujeitos por meio das manifestações culturais, simbólicas, do corpo e do movimento, a fim de vivenciar, compartilhar, desenvolver e transformar suas práticas corporais que caracterizam o processo de ensino-aprendizagem.

Neste sentido, os principais objetivos da Educação Física corroboram uma prática educativa alinhada com os desafios contemporâneos. Estes estão ligados a democratização do ensino, a busca de autonomia, reflexão crítica, e também traz para a pauta de discussões, a saúde e o lazer como possibilidades da Educação Física na escola (DARIDO \& RANGEL, 2005).

A cultura corporal inserida dentro destes objetivos pode guiar o educando para uma prática que vise: os princípios da inclusão, da diversidade, da preservação e da disseminação cultural, às vivências diferenciadas de práticas corporais, à autonomia e à formação crítico-reflexiva de suas ações na esfera da saúde e do lazer.

Desta maneira, a Educação Física pode estabelecer uma orientação na busca de ampliar o desenvolvimento global do educando e articulá-lo com o maior número possível de práticas e conhecimentos corporais, promovendo ações significativas importantes para a sua vida, de modo que possam integrar a corporeidade e o movimento à cultura corporal, isto "[...] implica, portanto, uma atuação intencional sobre o homem como ser corpóreo e motriz, abrangendo formas de atividades físicas, como a ginástica, o jogo, a dança e o desporto" (GONÇALVES, 1997, p. 134).

A Educação Física como componente curricular obrigatório da Educação Básica de acordo com a LDB - Lei de Diretrizes e Bases da Educação Nacional (BRASIL, 1996) ganhou visibilidade dentro do cotidiano escolar, pois, integrada ao projeto político pedagógico da escola, pode oferecer uma gama de conhecimentos para os educandos nos âmbitos cognitivos, motores, atitudinais e psicossociais, destacando-se principalmente com os conteúdos da cultura corporal. Nesse sentido, a LDB, em seu artigo primeiro, define que: 
A educação abrange os processos formativos que se desenvolvem na vida familiar, na convivência humana, no trabalho, nas instituições de ensino e pesquisa, nos movimentos sociais e organizações da sociedade civil e nas manifestações culturais. (BRASIL, 1996, art. $1^{\circ}$ )

Podemos entender que as manifestações culturais, na forma da lei, podem englobar os elementos da cultura corporal nos processos educativos no que tange à linguagem corporal, à construção de identidade, à socialização de saberes e às práticas culturais construídos historicamente, dentre outros aspectos relevantes para a prática dentro da Educação Física na escola.

Os Parâmetros Curriculares Nacionais - PCN's - ampliam ainda mais o debate sobre cultura corporal, no momento que afirma como direito de todos o acesso aos conteúdos e às capacidades que a Educação Física pode desenvolver, bem como o entendimento sobre o homem no contexto cultural e que este sujeito é capaz de produzir, reproduzir e transformar a cultura e a realidade.

A proposta dos Parâmetros Curriculares Nacionais adotou a distinção entre organismo - um sistema estritamente fisiológico - e corpo - que se relaciona dentro de um contexto sociocultural - e aborda os conteúdos da Educação Física como expressão de produções culturais, como conhecimentos historicamente acumulados e socialmente transmitidos. Portanto, a presente proposta entende a Educação Física como uma cultura corporal. (BRASIL, 1997, p. 25)

A Educação Física Escolar, pode oferecer ao educando uma gama de possibilidades que são expressas e estimuladas pela linguagem corporal, assim como o entendimento e a reflexão sobre as manifestações da cultura corporal, indo além de aulas meramente práticas, mas que possam dialogar com conteúdos conceituais e atitudinais, e ainda, com as necessidades e interesses dos mesmos.

A escola deve levar sempre em consideração os elementos que fazem parte da cultura corporal e também os que se referem à contemporaneidade, colaborando com a socialização dos conhecimentos e o aumento do padrão cultural dos educandos. Abaixo seguem algumas competências e habilidades propostas pelo PCN's que dizem respeito diretamente a cultura corporal as quais os educandos irão adquirir ao longo das aulas de Educação Física:

[...] refletir sobre as informações específicas da cultura corporal, sendo capaz de discerni-las e reinterpretá-las em bases científicas, adotando uma postura autônoma na seleção de atividades e procedimentos para a manutenção ou aquisição da saúde; (...) compreender as diferentes manifestações da cultura corporal, reconhecendo e valorizando as diferenças de desempenho, linguagem e expressão; (...) demonstrar autonomia na elaboração de atividades corporais, assim como capacidade para discutir e modificar regras, reunindo elementos de várias manifestações de movimento e estabelecendo uma melhor utilização dos conhecimentos adquiridos sobre a cultura corporal. (BRASIL, 1999, p. 42).

O professor pode oferecer ao educando uma grande diversidade de conteúdos e fazer com que o mesmo pense sobre as possibilidades diferenciadas e transformadas das manifestações corporais, dentro da base prática como também da teórica. A intervenção pedagógica do educador fundamentada na cultura corporal proporciona 
destaque ao respeito as diferenças regionais, culturais, simbólicas, corporais, e também, ao contexto contemporâneo em que ocorre a prática. Vale ressaltar que com o conhecimento sobre a cultura corporal, o educando terá competência e habilidade de apreciar as manifestacões corporais, posicionar-se crítica e reflexivamente perante os conteúdos aprendidos e discutidos em aula.

Nesse sentido, a abordagem cidadã, proposta pelos PCN's, se apresenta como uma forma de legitimar a cultura corporal em priorizar a cidadania, a autonomia, incentivar a criticidade, reconhecer e valorizar as diferenças e a pluralidade cultural, além de,

[...] promover o princípio da inclusão, com a inserção e a integração dos alunos na Cultura Corporal de Movimento, por meio de vivências que problematizem criticamente os conteúdos: jogos, esportes, danças, ginásticas, lutas e conhecimento sobre o corpo. (DARIDO \& RANGEL, 2005, p. 18)

Isto é, quando valorizamos e vivenciamos diferentes manifestações da cultura corporal - entendendo-as como jogo, esporte, dança, ginástica e lutas - estas que o homem produz e reproduz por meio da sua relação com a cultura e a natureza evidenciadas pela expressão corporal, podemos possibilitar uma transformação educativa, quando socializam e refletem criticamente sobre os conteúdos e externalizam em comportamentos de solidariedade e cooperação.

Uma aula de Educação Física pautada nos PCN`s possuem os elementos da Cultura Corporal de Movimento como seus objetivos e conta com as dimensões do conteúdo, entendo-os como conceituais, procedimentais e atitudinais, como base em seu processo de ensino-aprendizagem.
Com este panorama metodológico, a importância que se atribui ao movimento para a estruturação da cultura corporal é, de fato, desenvolver seus componentes nesta sociedade em transformação, que tem como grande objetivo educar o cidadão para o novo milênio, com uma visão crítica sobre o mundo.

Cabe ao professor de Educação Física problematizar, interpretar, relacionar, compreender com seus alunos as amplas manifestações da cultura corporal e, dessa forma, facilitar a aprendizagem de competências que levem o jovem e o adulto a analisar e sintetizar uma partida, um espetáculo de dança, a ocorrência de violência e a presença de anabolizantes no esporte, entre outros temas igualmente importantes. (BRASIL, 1999, p. 146)

É necessário que o professor de Educação Física encontre identidade em sua área de estudo, fundamental para que faça seu educando compreender o seu papel como: produtor, reprodutor e transformador de cultura; reconhecedor dos conhecimentos produzidos e desfrutados pela sociedade assim como práticas corporais juntamente com sua diversidade, linguagens e pluralidade.

Desta maneira, a Educação Física Escolar amplia a visão sobre a cultura corporal, trazendo reflexões sobre valores, comportamentos e vivências de diferentes práticas e linguagens corporais e as influências e as contribuições da cultura local e global, e também, para aumentar a produção e o repertório de conhecimentos para a vida dos educandos.

Juntamente com o conhecimento sobre a cultura corporal, é importante percebemos que este conteúdo - dança, jogo, ginástica, esportes, lutas, conhecimento 
sobre o corpo, etc. - está relacionado com uma grande teia que irá abarcar aspectos que estão envolvidos com estes respectivos conteúdos.

Neste momento, serão discutidos alguns conceitos e reflexões em torno dos pilares da educação propostos pela UNESCO e a tipologia dos conteúdos e objetivos de ensino apresentados por Zabala (1998), para que possamos entender a relação com a cultura corporal que se faz presente na Educação Física Escolar.

Zabala (1998) traça um caminho para abordar os conteúdos na escola e classifica-os em conceituais, procedimentais e atitudinais, demonstrando a função social do ensino e sua relação com os tipos fundamentais de aprendizagem, juntamente com as experiências vivenciadas pelos alunos em sua realidade.

Essa tipologia está diretamente elencada com os pilares da educação - aprender a conhecer, aprender a fazer, aprender a viver juntos e aprender a ser - formando as bases estruturais da educação na atualidade. E vão se caracterizar por ser instrumentos de compreensão, de troca de experiências, de solução de conflitos e de desenvolvimento global, respectivamente.

Para o autor, o processo de ensino-aprendizagem envolve aspectos objetivacionais, interativos e afetivos que envolvem a escola e os educandos. No momento em que aborda o conteúdo, suas concepções estão além do que ensinar, mas em uma perspectiva maior, o por que ensinar, e assim, a classificação dos conteúdos se ligam diretamente com os objetivos educacionais. Na classificação dos conteúdos surgem as problemáticas que englobam a aprendizagem dos conhecimentos: o que se deve aprender? (factual e conceitual), o que se deve fazer (procedimental) e como se deve ser? (atitudinal).

As características dos conteúdos são conceituadas de acordo com Zabala (1998) para a construção de uma aprendizagem de forma mais global e que preconizem as dimensões do ser humano, são elas: factuais " [...] se entende o conhecimento de fatos, acontecimentos, situações, dados e fenômenos concretos e singulares" (p.41); conceituais "[...] se referem ao conjunto de fatos, objetos ou símbolos que têm características em comum" (p. 42); procedimentais "[...] são um conjunto de ações ordenadas com um fim" (p. 45); atitudinais "[...] valores, normas e atitudes, para reflexão sobre pontos positivos e negativos de determinado modelo de comportamento"(p.46).

Os professores ao formular seu planejamento devem se ater as referências e experiências extra-escolares dos educandos, as particularidades e diversidades culturais, as aprendizagens significativas, desafiantes e motivadoras. Para isso, os Parâmetros Curriculares Nacionais (PCN) abordam a articulação dos pilares da educação com as propostas educacionais para professores na sociedade contemporânea:

A preocupação em contextualizar competências, habilidades e conteúdos - e, nestes, os conceitos, explicitando seu significado, evidenciando sua importância e identificando sua articulação com o todo - propõe-se, sobretudo, a propiciar e estimular a reflexão do professor, a quem se destina este documento. Caberá a ele, no cenário de sua experiência cotidiana, opinar a respeito da validade dos recortes efetuados. (BRASIL, 1999, p.24)

Neste sentido, Zabala (1998) contribui com a posição do professor nos dias 
de hoje, principalmente quando enfatiza as características de um bom profissional, que seria: buscar competência seu trabalho, analisar a sua prática profissional, interpretar suas ações no ambiente de ensino-aprendizagem.

De uma maneira prática, poderíamos compreender essas sequências e as características dos conteúdo de acordo com sua tipologia, com destaque para o conhecimento prévio do educando, o desenvolvimento de forma contributiva, lúdica e planejada, e também em atividades desafiadoras, motivantes e autônomas.

E ainda, ressalta que a função primordial da escola é gerar a formação integral do educando, por meio de vivências abrangentes tanto dentro como fora da escola, para que formem um liame, de modo que possam conhecer e se relacionar com o quê e com quem está a sua volta e exercer a sua cidadania. Isto é, o processo de ensino-aprendizagem e a relação professor-educando deve expor uma relação intencional, responsável e interativa que se compreenda em uma relação pedagógica transformadora e com valores educativos.

Assim, para que se possa compreender a real preocupação com o contexto educacional nesta teia de relações, os PCN's ressaltam a importância da junção de diferentes concepções em prol da "[...] busca de uma Educação Física que articule as múltiplas dimensões do ser humano" (BRASIL, 1997, p.5), ou seja, que integre ao seu currículo às dimensões do conteúdo, pautadas nos pilares da educação.

\section{CONSIDERAÇÕES FINAIS}

Verifica-se a importância de se trabaIhar com as três dimensões de forma integrada juntamente com os pilares da educação, privilegiando todas as fases e características salientadas, integrando os componentes curriculares, bem como o favorecimento de uma prática critico-reflexiva e voltada para a cidadania, pautadas nos pilares da educação, de forma permanente na vida do educando, que só tem a contribuir com a Educação Física Escolar.

Não se pode deixar de salientar a relação entre a cultura corporal, os pilares da educação e a classificação do conteúdo, que apresentam uma íntima conexão, no momento em que os conteúdos da cultura corporal podem ser trabalhados em toda a sua extensão: no aprender a conhecer, a fazer, a ser e a viver, bem como nas dimensões conceituais, procedimentais e atitudinais.

A triangulação docente-discente-conteúdo deve ser interativa e participativa, para que haja reflexão sobre a ação da natureza dos conteúdos, bem como com os participantes diretos deste processo, pensando sempre na diversificação do conteúdo programático, reunindo os componentes da cultura corporal, estabelecendo desafios, reflexões e compreensão da realidade.

Sobre os pilares da educação, a direta ligação com a cultura corporal se encontra na compreensão dos fatos e dos comportamentos, na troca de experiências, no aprendizado de resolução de conflitos, sobre o conhecimento de outras culturas e também em valorizar a cultura, se desenvolvendo de maneira global, ou seja, um aprendizado que aproxime o educando do que está acontecendo ou o que aconteceu globalmente, aumento seu conhecimento sócio-histórico-contemporâneo, se podemos cunhar desta maneira. 
Em sua aplicação para a dimensão dos conteúdos relacionadas com os componentes da cultura corporal é fundamental conhecer as transformações na sociedade, vivenciar e adquirir fundamentos básicos das práticas corporais, valorizar o patrimônio das manifestações culturais e a dimensão do conviver.

É essencial que a cultura corporal desenvolva a multiplicidade dos conteúdos e de suas práticas, assim como, a inclusão dos educandos dentro dos temas que englobam o jogo, a dança, o esporte, a luta, a ginástica e o conhecimento sobre o corpo em conjunto com os objetivos educacionais da dimensão dos conteúdos, com ênfase nas experiências vividas e o que eles podem construir ao longo do processo de ensino-aprendizagem.

Em resposta a problemática deste ensaio, o professor deve, primeiramente, saber o porquê de ensinar, procurando trabalhar baseado nos pilares da educação, diversificando o máximo os conteúdos no currículo da Educação Física, a fim de ampliar o repertório cognitivo, atitudinal e motor dos educandos e aquilo que se pretende alcançar de modo fundamentado e orientado.

Assim, os elementos da cultura corporal podem subsidiar este processo, formando o educando para lidar com as diferentes manifestações corporais, oferecendo arcabouço necessário para que o mesmo possa ter atitudes críticas e reflexivas na sociedade em que vive, e consequentemente, possa transformá-la. Isto é, no sentido de uma prática corporal humanizadora, com a apreciação e a compreensão das concepções, relações e manifestações da cultura corporal.
Este conhecimento sustentado na cidadania, na construção do conhecimento e em uma ampla visão de mundo, faz com que o professor transmita o conhecimento não de uma forma imediatista e reprodutora, mas sim, de maneira planejada, estruturada, interativa e responsável, de modo que a triangulação discente-docente-conteúdo tenham uma base sólida, construída em cima de valores, princípios, e indo ao encontro da teoria de Zabala (1998) com as dimensões do conteúdo. Chegando a conclusão e compreendendo o indivíduo, o conhecimento e os objetivos educacionais como uma única e entrelaçada teia. Com esta visão, todo o processo de ensino-aprendizagem e de prática pedagógica farão realmente sentido.

Assim, os conteúdos da cultura corporal dentro da Educação Física Escolar poderão ser devidamente apreciados, vivenciados e contextualizados na realidade sócio-cultural dos educandos, de uma maneira que eles possam analisar e abordar essa teia educacional de significados em torno do que se aprende no ambiente escolar e do que se vive dentro e fora dele.

\section{REFERÊNCIAS}

BRASIL, Ministério da Educação e do Desporto. Lei de Diretrizes e Bases da Educação Nacional. Lei n. ${ }^{0}$ 9.394/96, de 20 de dezembro de 1996.

BRASIL, Secretaria de Educação Fundamental. Parâmetros curriculares nacionais: Educação física. Volume 7. Brasília: MEC/SEF, 1997.

BRASIL, Secretaria de Educação Média e tecnológica. Parâmetros curriculares nacionais: Ensino Médio: Linguagens, códigos e suas tecnologias. Brasília: MEC/SEMTEC, 1999. 
DAOLIO, J. Educação Física e o conceito de cultura. 2a ed. Campinas: Autores Associados, 2007.

DARIDO, S. C. \& RANGEL, I. C. A. Educação Física na escola: implicações para a prática pedagógica. Rio de Janeiro: Guanabara Koogan, 2005.

GEERTZ, Clifford. A interpretação das culturas. 1 . ed., 13 reimpr. Rio de Janeiro: LTC, 2008.

GONÇALVES, M. A. S. Sentir, pensar, agir: corporeidade e educação. Campinas: Papirus, 1997.
HOUAISS, Antônio. Dicionário da Língua Portuguesa. Rio de janeiro: Objetiva, 2007.

RANGEL, I. C. A. et al.. Educação Física Escolar e Multiculturalismo: possibilidades pedagógicas. Motriz, v. 14, n.2, 2008.

ZABALA, Antoni. A prática educativa: como ensinar. Porto Alegre: Editora Artes Médicas Sul Ltda., 1998.

\title{
A DIALOGUE ON THE BODY CULTURE AND DIMENSIONS OF CONTENT WITHIN A WEB OF RELATIONSHIPS
}

\begin{abstract}
This article aims to look for a reflection on the relationship of body culture and its application in Physical Education within the dimensions of content and the pillars of education in teaching-learning and pedagogical practice. Questioning if the physical education teacher, dealing the contents of body culture could have immediate knowledge to develop the capabilities of students in a way they find meaning in what they do? Thus, there is the importance of understanding the elements of the educational process and also its objectives and content as a true educational web.
\end{abstract}

Keywords: Body culture; Dimension of contents; Teaching-learning process; Pedagogical Practice. 